\title{
CLOSE BINARY MODELS FOR LUMINOUS BLUE VARIABLE STARS
}

\author{
J. S. GALLAGHER \\ Lowell Observatory \\ 1400 West Mars Hill Road \\ Flagstaff, Arizona 86001 \\ U.S.A.
}

\begin{abstract}
The evolution of massive close binary stars inevitably involves mass exchange between the two stellar components as well as mass loss from the system. A combination of these two processes could produce the stellar wind-modulated behavior seen in LBVs. The possibility that LBVs are powered by accretion is examined, and does not appear to be a satisfactory general model. Instead, identification of LBVs with close binaries in high mass-loss rate or common envelope evolutionary phases shows promise.
\end{abstract}

\section{Introduction}

This conference has raised a variety of unanswered questions concerning luminous blue variable stars (hereafter LBVs). What physical class of star are they? Why does an unstable phase occur for only a fraction of evolved very luminous stars? How does the instability process operate and for how long? In this review I briefly consider one set of answers; i.e., that some LBVs are produced by the evolution of massive binary stars.

Invocation of binary star evolution is justified by prior experience when dealing with unusual stellar characteristics that apply only to a subset of stars in a given physical class. Examples of successful applications of binary models to peculiar classes of stars include classical, recurrent, and dwarf novae; symbiotic stars: barium stars; and luminous x-ray stars. But there is a price to be paid when including binaries. The evolutionary function $E$ of a single massive star can be represented to zero order as $E\left(M_{0}, t, z, \dot{M}_{w}(t), \vec{L}(t), \bar{B}(t)\right)$, where $M_{0}$ is the initial mass, $z$ the mean metallicity, $\dot{M}_{w}(t)$ the wind mass-loss rate, $\vec{L}$ the angular momentum, and $\vec{B}$ the average magnetic field strength.

This is bad enough! However, the evolutionary function for binaries is even more complex as all of the above terms now apply to both members of the binary, and additional terms relating to orbital period, mass ratio time, and accretion rates (all functions of time) must be added (e.g., Eggleton 1985). The daunting complexity of binary evolution has caused some people to decide that this problem is simply a cleverly disguised opportunity for theorists (and enterprising observers) to ascend into free-parameter heaven. This view, whatever its merits, ignores the fact that many massive stars are members of binaries where interactions must occur during the normal course of evolution.

Several arguments suggest that binary evolution is relevant to the existence of LBVs: (1) At least $40 \%$ of massive stars are born in binary star systems with some potential for 
binary interaction during their lifetimes (cf. Garmany et al. 1980). (2) There is a proven tendency for interacting binary stars to experience episodic outbursts with mass ejection from the system. (3) The lowered gravitational potentials at the surfaces of near Rochelobe filling members of binary systems should lead to enhanced mass-loss rates even during quiescent phases. (4) The evolution of binary stars takes place over longer time scales than those of single stars of similar mass. These features imply that processes due to the presence of massive binaries will affect only part of the massive star stellar population, are likely to be associated with episodic mass ejections and high mass-loss rates, and may occur in older environments than those where massive single stars are found. Binary stars must be included in possible models for LBVs, as LBVs share all of the above symptoms of ongoing binary star evolution.

\section{Accretion-Powered Binary Model}

Theoretical models and observations both support the view that mass ejection events and bolometric luminosity variations can be produced by accretion within binary star systems. This aspect of binary systems is perhaps most clearly illustrated by outbursts in symbiotic stars, where disk accretion is often the physical cause of eruptive behavior (cf. Kenyon 1986).

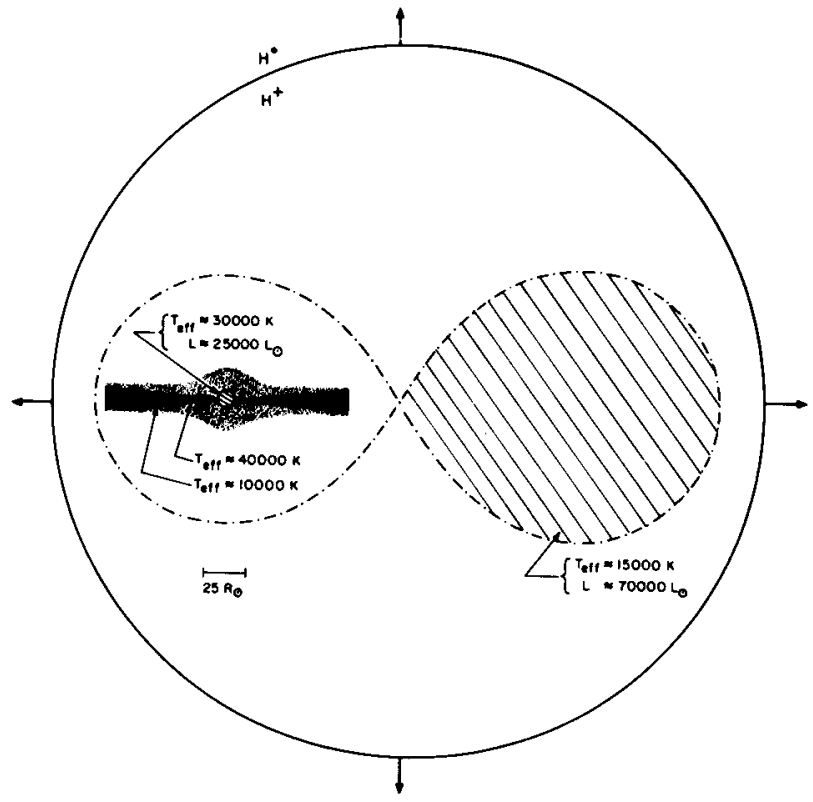

Figure 1. Schematic model of a massive binary system with disk accretion (Kenyon and Gallagher 1985).

In a key paper, Bath (1979; see also Webbink 1979) suggested that the accretion model be extended to the LBVs. The disk accretion model is schematically illustrated in Figure 1, which is reproduced from Kenyon and Gallagher (1985). Bath assumed an $\alpha$ 
disk to estimate temperatures of the accreting system and used simple potential energy arguments to derive an accretion luminosity $L_{a}$,

$$
L_{a} \approx \frac{G M \dot{M}_{a}}{R} \approx 10^{6}\left(\frac{v_{e s c}}{1000}\right)^{2} \frac{\dot{M}_{a}}{\left(6 \times 10^{-3}\right)} L_{\odot} .
$$

Here $v_{e s c}$ is in $\mathrm{km} \mathrm{s}^{-1}$, and $\dot{M}_{a}$ is in units of $M_{\odot} \mathrm{yr}^{-1}$. The last term in this expression is cause for concern. Unless a compact object is allowed such that $v_{\text {esc }} \gg 600 \mathrm{~km} \mathrm{~s}^{-1}$, then very high accretion rates are required to achieve luminosites in the LBV range.

A compact accretor, however, is unlikely to power most in LBVs. The minimum mass of the accreting object can be estimated from the Eddington luminosity $L_{E}=3 \times$ $10^{4}\left(M / M_{\odot}\right) L_{\odot}$, where $M$ is the mass of the accreting star. For an LBV luminosity of $L_{\text {bol }} \geq 3 \times 10^{5} L_{\odot}$, the accreting star should have a mass of $M \geq 10 M_{\odot}$, which exceeds the masses of all but the most extreme stellar black holes.

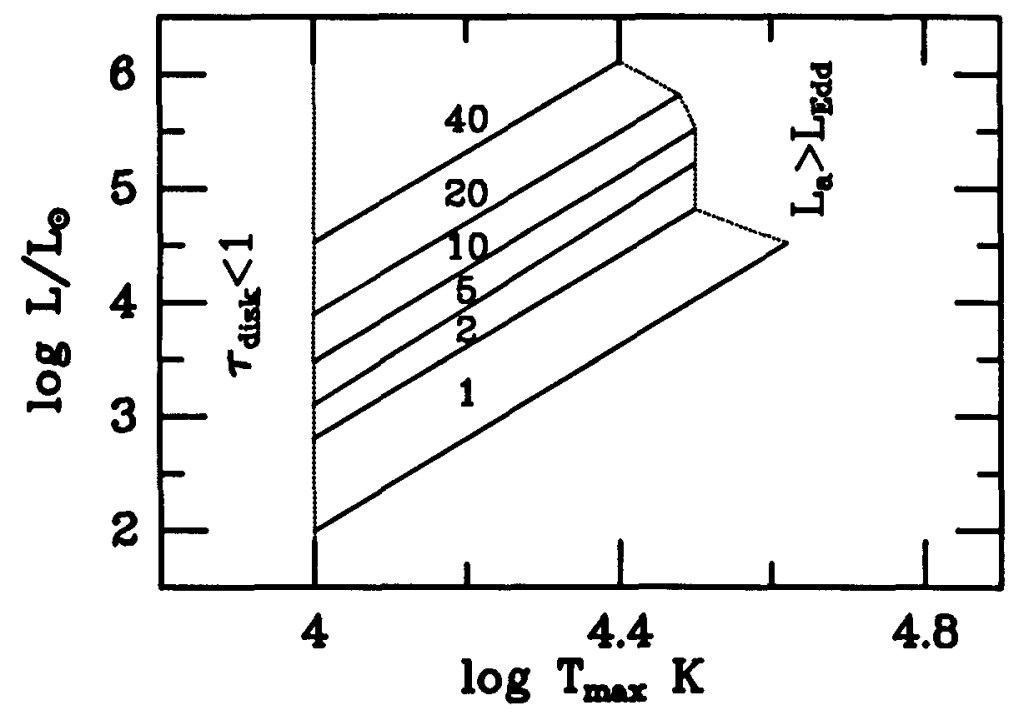

Figure 2. Luminosity-temperature curves for Bath (1979) accretion models. The tracks are labeled by the mass of the accreting star. A lower bound is set when the disk becomes optically thin and the upper bound by $L_{a}>L_{E}$.

A second difficulty is illustrated in Figure 2, which is based on Bath (1979). For a system in which the accretion disk is a major luminosity source, temperature will increase with $\dot{M}_{a}$; e.g., in Bath's model $T_{\max } \propto \dot{M}_{a}^{\gamma}$ with $\gamma \approx 0.25$. The behavior of this model is inconsistent with the observations showing that $\mathrm{LBV}$ s reach maximum visual luminosity at minimum temperature and the evidence for roughly constant $L_{\text {bol }}$ in LBVs. These properties are most readily understood in terms of variations in $\dot{M}_{w}$ which effect the location of the pseudophotosphere and thus $L_{v i s}$, behavior which is well-documented in the early post-maximum development of classical novae (Gallagher and Code 1974; Ruggles and Bath 1979).

Disk accretion models do not seem to fully explain LBVs. Thus Figure 1 might be representative of a quiescent phase LBV where stellar photospheres and the disk all 
contribute to the visual luminosity, while the disk is also responsible for extra line emission. Detailed comparisons with observations are necessary to see if even this scaled-down disk accretion model is acceptable.

An alternate version of this model is possible if LBVs are experiencing saturated accretion; i.e., if $L_{a} \geq L_{E}$. We then can ask whether the wind would carry the excess $L_{a}$, as suggested by Żytkow's (1973) and Kato's (1985) models. The Eddington limit provides a natural reason for near constant $L_{\text {bol }}$ from the system, and fluctuations in $\dot{M}_{w}$ are likley to occur and produce the desired visual light variations. We could consider these types of objects as "stealth" binaries, since the binary nature of the underlying system is largely but not completely optically obscured by a pseudophotosphere associated with the accreting star.

We require very high accretion rates of $\dot{M}_{a} \geq 6 \times 10^{-3} M_{\odot} \mathrm{yr}^{-1}$ to produce a hidden binary from a system where the accretor is a main-sequence star. It is fair to ask if such high rates can be expected in nature. Webbink (1985) has emphasized that there are three natural time scales associated with the mass-losing star which can drive accretion rates in binaries. As a rough estimate of allowable rates for each time scale $\tau_{i}$ from a star of mass $M_{0}$ with available mass fraction $f$ for mass loss, we have $\dot{M}_{a}(i) \approx f M_{0} \tau_{i}^{-1}$. Even for a massive star, nuclear time scales are $\geq 10^{6} \mathrm{yr}$, so we expect $\dot{M}_{a}(n u c) \leq 10^{-4} M_{\odot} \mathrm{yr}^{-1}$. Thermal time scales are similar, so we must rely on the dynamical time scale to produce Eddington-critical accretion rates. Accretion on dynamical time scales occurs when a star cannot readjust to the changes in Roche lobe size in an interacting binary, and in principle can produce the required high values of $\dot{M}_{a}$.

A detailed study of this problem is in progress as M. S. Hjellming's Ph.D. thesis at the University of Illinois (see Hjellming and Webbink 1987). However, stars with convective envelopes are known to be unstable against dynamical mass exchange, and thus a cool supergiant donor is a good candidate for the model. Possibly stars near the Eddington limit are also sufficiently unstable to make this mechanism work (cf. McClusky and Kondo 1976). A problem that remains to be resolved is the response of the accreting star to rapid accretion. If the accreting star responds by expanding into contact or near contact, then accretion will presumably cease and the model fails (see Kippenhahn and MeyerHoffmeister 1977; Webbink 1985).

A best model for an accretion-powered, binary LBV has the following properties: (1) The total initial mass of the system must be $\geq 20 M_{\odot}$. (2) The orbital period must be long enough to allow an evolved primary with a convective envelope to be present ( $P \geq 100$ days). (3) An accreting main-sequence $B$ star is normally optically obscured by a massive wind powered by the accretion process. This picture is consistent with an R81 type of object (Stahl et al. 1987), and possibly the related B[e] stars (cf. Shore et al. 1987; Zickgraf et al. 1988).

However, the accretion-powered binary model has major flaws and is unlikely to provide a general explanation of LBVs: (1) At super-Eddington $\dot{M}_{a}$ levels, the estimated lifetimes of $\approx 10^{3} \mathrm{yr}$ are too short. (2) Fine tuning is needed to achieve near constant $L_{\mathrm{bol}}$. (3) The response of the accreting star may further limit the duration of this evolutionary phase. 


\section{Binary Stars and Enhanced Mass-Loss Rates}

Binary stars may produce LBVs due to their ability to support high mass-loss rates rather than as a result of high accretion luminosities. This type of model is particularly attractive when one is considering whether a close binary might mimic a very massive single star. For high-mass stars, the bolometric luminosity scales almost linearly with initial mass, $L_{n u c}\left(M_{1}+M_{2}\right) \approx L_{n u c}\left(M_{1}\right)+L_{n u c}\left(M_{2}\right)$. A binary star with initial component masses $M_{1}$ and $M_{2}$ therefore will be nearly indistinguishable from a single star with initial mass $M_{1}+M_{2}$ in terms of nuclear luminosity, and can be even more luminous than the single star if accretion and related processes contribute radiative luminosity. Binaries with initial total masses exceeding about $60 \mathrm{M}_{\odot}$ will have the nuclear luminosities of LBVs for mass ratios near unity, and such systems are known to exist (e.g., Garmany et al. 1980; Hilditch and Bell 1987). Indeed, as A. V. Tutukov (private communication and see Tutukov and Yungel'son 1980) has emphasized, binary systems with initial total masses of $\geq 60 \mathrm{M}_{\odot}$ are likely to occur at least as frequently as single stars of similar mass in the Galactic OB stellar population.

\subsection{Mass Loss from Main-Sequence Close Binaries}

The primary star in a close binary will inevitably expand in radius as a result of nuclear evolution and may fill its Roche lobe during (Case A) or soon after (Case B) the main-sequence core hydrogen-burning evolutionary phase (cf. Vanbeveren et al. 1979; Vanbeveren 1982; Nakamura and Nakamura 1984). The models suggest that the ensuing mass transfer to the secondary is likely to lead to a contact binary and extensive mass loss from the system before mass exchange is complete (see also Ziólkowski 1979). This process may be further enhanced in high-mass binaries by the effects of radiation pressure (McCluskey and Kondo 1976). The precise period ranges and other details of the process are not fully established (e.g., the lower period bound for Case $\mathrm{A}$ in a given system depends on stellar mass-loss rates and degree of convective core overshooting; Doom 1984; Sybesma 1985, 1986a,b), and quantitative predictions about frequencies of occurrence are thus not available.

The qualitative aspects of this process, however, are interesting in the present context. As discussed above, the necessary luminosity is supplied by nuclear burning in the two binary members for $M(1+2) \geq 60 M_{\odot}$. Mass exchange rates during Roche lobe overflow can be very high, $>10^{-4} M_{\odot} \mathrm{yr}^{-1}$ in massive binaries. Thus if the mass lost from the system is even a modest fraction of the mass exchange rate, $\dot{M}_{w} \approx 10^{-4} M_{\odot} \mathrm{yr}^{-1}$ can be achieved. This level of $\dot{M}_{w}$ will produce a pseudophotosphere that is larger than the dimensions of a short-period massive binary for LBVs near maximum light (see Vanbeveren et al. 1979; Gallagher et al. 1981). Mass loss from the primary should also lead to the evidence for CNO-cycle processing that is found in some LBVs (Tutukov and Yungel'son 1983). A further bonus has been noted by Tutukov and Yungel'son (1980) in that this process will yield objects which appear as the most massive "single" stars due to the mass gained by the original secondary component.

The existence of semidetached and contact $\mathrm{O}$ star binaries with both components on or near the main-sequence (e.g., Hilditch and Bell 1987; Leung 1988) shows that this sequence of events has the potential to occur in nature. Additional work is needed to see if the specifics of the mass-loss process and other aspects of this model quantitatively fit observed properties of the LBVs. 


\subsection{Common Envelope Binaries}

In massive binary systems with compact components, the less evolved member can readily supply sufficient $\dot{M}_{a}$ such that $L_{a}>L_{E}$. Under these circumstances a common envelope will surround both components of the binary. The envelope is driven by dynamical effects and can carry off large amounts of matter and angular momentum from the system (e.g., Ostriker 1975; Paczynski 1976). The existence of short-period binaries containing compact objects is direct evidence of the importance of the common envelope phase in the evolution of massive binary star systems (e.g., Tutukov and Yungel'son 1979).

The resulting buried binary will appear as an object with a pseudophotosphere whose radius is controlled by $\dot{M}_{w}$ and is therefore a potential LBV candidate. The luminosities of common envelope binaries can lie in the observed range for LBVs during the initial phase of the interaction between components, which may last for a period of $\approx 10^{3}-10^{4} \mathrm{yr}$ (e.g., Taam et al. 1978). An additional signature of this and other binary ejection processes is expected to be an asymmetrical envelope which lies preferentially in the equatorial plane of the binary system (Bodenheimer and Taam 1984; Livio and Soker 1988). The ejection velocities for this process may also be in the appropriate range for LBVs.

\subsection{Musings on $\eta$ Carinae}

Since $\eta$ Carinae is in an area rich in young, massive stars, it stands out as an excellent single star LBV candidate resulting from an instability in an extremely massive star. However, the possible presence of bipolar structure in the ejecta reviewed by $K$. Davidson (this volume) suggests that an effort should be made to consider a binary model for this most extraordinary LBV.

In this spirit, we can ask what type of event in a binary could lead to an outburst of the magnitude seen in the 19 th century in $\eta$ Carinae? One interesting model that might merit further exploration is a merger between components in a young binary (or multiple star?) system. The thermal energy generated in this type of event should be sufficient to account for both the radiated power and ejection of several $\mathrm{M}_{\odot}$ of material with bipolar symmetry. Is it then possible that $\eta$ Carinae is an extreme example of the Tutukov mass exchange mechanism operating in a high-mass binary system yielding a single superstar?

\section{Conclusions}

1. Accretion-powered massive binary models for LBVs as a class require tricky finetuning and are unlikely to provide a general explanation for LBVs.

2. Enhanced mass loss can occur in high-mass binary systems during mass exchange and common envelope evolutionary phases. This will produce a superwind which is the key physical characteristic of LBVs. Radiative luminosities of $10^{5}-10^{6} \mathrm{~L}_{\odot}$ can be provided by a combination of nuclear and dynamical sources within known examples of such classes of binary stars. In addition to naturally producing most of the observable characteristics of LBVs, this class of model also provides a natural distinction between LBVs and hypergiants of similar luminosity which do not show the LBV phenomenon.

3. The atmospheric characteristics of LBVs resemble those seen in superwinds from known binary systems (e.g., symbiotic stars). Thus the qualitative argument can be made that binary LBV model will have the desirable propertites of high $\dot{M}_{w}$, low $v_{\infty}$, bipolar 
ejection, complex spectral line profiles, interesting emission lines (e.g., lots of [Fe II]), and optical variability.

4. We should consider the possibility that the $\eta$ Carinae outburst was associated with an unusual event in a young binary or multiple star system.

\section{Acknowledgments}

I would like to thank my colleagues Otto Franz, Icke Iben, Scott Kenyon, Sasha Tutukov, and Ron Webbink for providing me with an appreciation for the richness of binary evolution. This paper has been supported in part by the Lowell Observatory Research Fund. Travel support from the IAU is also gratefully acknowledged.

\section{References}

Bath, G. T. (1979). Nature 282, 274.

Bodenheimer, P. and Taam, R. E. (1984). Ap. J. 280, 771.

Doom, C. (1984). Astron. Ap. 138, 101.

Eggleton, P. H. (1985). In Interacting Binary Stars, J. E. Pringle and R. A. Wade (eds.), Cambridge University, Cambridge, p. 21.

Gallagher, J. S., and Code, A. D. (1974). Ap. J. 189, 303.

Gallagher, J. S., Kenyon, S. J., and Hege, E. K. (1981). Ap. J. $249,83$.

Garmany, C. D., Conti, P. S., and Massey, P. (1980). Ap. J. 242, 1063.

Hilditch, R. W., and Bell, S. A. (1987). Astron. Ap. 176, 59.

Hjellming, M. S., and Webbink, R. F. (1987). Ap. J. 318, 794.

Kato, M. (1985). Pub. Astr. Soc. Japan 37, 19.

Kenyon, S. J. (1986). The Symbiotic Stars, Cambridge University, Cambridge.

Kenyon, S. J., and Gallagher, J. S. (1985). Ap. J. 290, 542.

Kippenhahn, R., and Meyer-Hoffmeister, E. (1977). Astron. Ap. 54, 539.

Leung, K.-C. (1988). In Critical Observations vs. Physical Models for Close Binary Systems, in press.

Livio, M., and Soker, N. (1988). Ap. J. 329, 764.

McClusky, G. E., and Kondo, Y. (1976). Ap. J. 208, 760.

Nakamura, M., and Nakamura, Y. (1984). Ap. Sp. Sci. 104, 367.

Ostriker, J. P. (1975). Paper presented at "The Structure and Evolution of Close Binary Systems," IAU Symposium No. 73.

Paczynski, B. (1976). In The Structure and Evolution of Close Binary Systems, IAU Symposium No. 73, P. Eggleton, S. Mitton, and J. Whelan (eds.), Reidel Publishing Co., Dordrecht, p. 75.

Ruggles, C. N., and Bath, G. T. (1979). Astron. Ap. 80, 97.

Shore, S. M., Sanduleak, M., and Allen, D. A. (1987). Astron. Ap. 176, 59.

Stahl, O., Wolf, B., and Zickgraf, F.-J. (1987). Astron. Ap. 184, 193.

Sybesma, C. H. B. (1985). Astron. Ap. 142, 171.

. (1986a). Astron. Ap. 159, 108.

(1986b). Astron. Ap. 168, 147.

Taam, R. E., Bodenheimer, P., and Ostriker, J. P. (1978). Ap. J. 222, 269.

Tutukov, A., and Yungel'son, L. (1979). In Mass Loss and the Evolution of O-Type Stars, IAU Symposium No. 83, P. S. Conti and C. W. H. de Loore (eds.), Reidel Publishing Co., Dordrecht, p. 401. 
. (1980). Pis'ma Astron. Zh. 6, 491 (Sov. Astron. Lett. 6, 271, 1981).

. (1983). Pis'ma Astron. Zh. 9, 230 (Sov. Astron. Lett. 9, 124).

Vanbeveren, D. (1982). Astron. Ap. 105, 260.

Vanbeveren, D., Grève, J. P., van Dessel, E. L., and de Loore, C. (1979). Astron. Ap. 73, 19.

Webbink, R. F. (1979). In Changing Trends in Variable Star Research, IAU Colloquium

No. 46, F. M. Bateson, J. Smak, and I. H. Ulrich (eds.), University Waikato, Hamilton,

New Zealand, p. 102. - (1985). In Interacting Binary Stars, J. E. Pringle and R. A. Wade (eds.),

Cambridge University, Cambridge, p. 39.

Zickgraf, F.-J., Wolf, B., Stahl, O., and Humphreys, R. M. (1988). Preprint.

Ziólkowski, J. (1979). In Mass Loss and Evolution of O-Type Stars, IAU Symposium No.

83, P. S. Conti and C. W. H. De Loore (eds.), Reidel Publishing Co., Dordrecht, p. 385. Żytkow, A. (1973). Acta Astr. 23, 121.

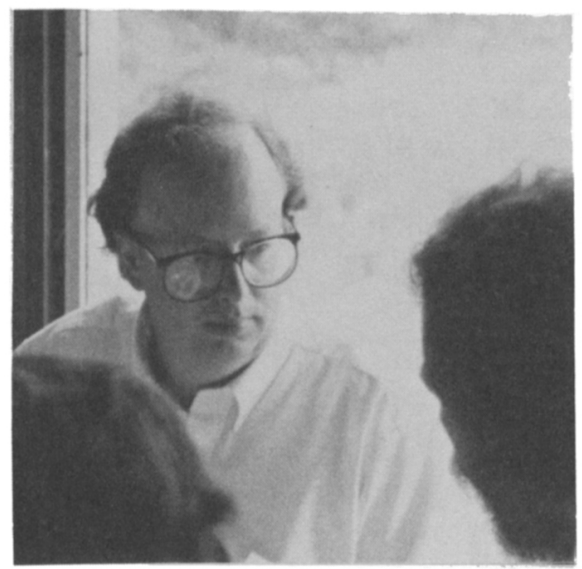

Jay Gallagher 


\section{DISCUSSION}

Wolf: The best way to study the physical nature of LBV's is perhaps to look at them at minimum. R71 at quiescence was modeled by the Munich group; they could fit the observed continuum fairly well with the continuum of an early B-supergiant model. Is this finding consistent with any of your binary scenarios?

Gallagher: In a qualitative sense, yes. Since a binary can be embedded in a common envelope and/or in an optically thick wind, it is hard to distinguish the nature of the underlying energy source. Perhaps the binary model would be characterized by a non-spherical atmosphere or by effects associated with lower gravity? Maybe Dr. Kudritzki could comment on this issue.

Kudritzki: I don't know. One problem I encountered with my hydrostatic NLTE energy-distribution-fitting of R 71 was that the NLTE model with the correct gravity $(\log g \approx 1.6)$ shows a Balmer jump that is not observed. An extended pseudo-photosphere might be better. But without a calculation it is hard to predict.

De Groot: Since Vanbeveren's remark about a spiralling-in companion star yesterday, I have been wondering about binaries and LBV's. On each day of this meeting we have heard about difficulties with ejected shells and their expansion velocities, [NII] profiles that do not look like outburst shells or red supergiant ejecta ... Binaries have the potential of solving these problems. The cartoon on your last viewgraph resembles Paresce's picture of AG Car.

Fried jung: One must be careful not to mix up classical novae, symbiotic stars, and LBV's. There are good reasons for believing the existence of optically thick winds for classical novae. For symbiotic stars, however, there is not so much evidence for this. Some are thought to contain a red giant and an accreting main sequence star. According to the present orthodoxy (which might change), sometimes there is an accreting white dwarf with surface nuclear reactions. Such a star would expand and move in the $\mathbf{H}-\mathrm{R}$ diagram along a track like that of a planetary-nebula-nucleus but in the opposite direction. A wind from such an expanded white dwarf would resemble that from a normal hot star.

Gallagher: Certainly one has to be careful about mixed metaphors when comparing LBV's with novae and symbiotic stars. Still, in most of these eruptive variables the optical properties are largely controlled by stellar winds, i.e., supersonic mass flows through a pseudo-photosphere, and in this respect they are similar to LBV's.

Zickgraf: The merger model for $\mathrm{B}[\mathrm{e}]$ stars is very interesting. But isn't there a problem with the statistics? Presently we know $7 \mathrm{~B}[\mathrm{e}]$ supergiants in the LMC. Would you expect to have so many mergers?

Gallagher: A merger would be a short-lived phase that is accessible to only a fraction of massive binaries. To make an estimate, we need better statistics on massive binaries in the LMC.

Conti: Almost the only person doing such work on the LMC is Virpi Niemela. She has found a handful of short-period O-type binaries. This work requires considerable observing time and much encouragement. 
Appenzeller: Concerning the suggestion that an LBV is a mass-exchange binary containing (as a donor star) a red supergiant with an extended outer convection zone: According to the classical investigations by Paczynski and his group and by Plavec, even with hydrodynamic effects taken into account, in such systems the mass exchange takes place on a time-scale not much larger than the dynamical time-scale. Even with optimistic assumptions, the lifetime of such a state should not exceed a few years. This is much shorter than the empirical lifetime of an LBV as derived, e.g., from kinematic ages of circumstellar matter.

Gallagher: I agree with your comment. The estimated lifetime of 1000 years that I mentioned may too optimistic, and even that is too short to completely explain LBV's.

Davidson: At least some LBV's appear to have bipolar morphologies; the easiest ways to get suitable axes are either by rotation or by orbiting; and yet many of us at this meeting seem uncomfortable with either rotation or binaries (because we'd like to minimize the number of parameters, I suppose). However, if we choose to do so, a fairly close binary companion can be employed in a way that is less ambitious than Gallagher's and Vanbeveren's scenarios: we could use the companion star merely as a device to enforce co-rotation. Its orbital speed, after all, is unaffected by the radiation pressure that reduces effective gravity in the LBV star's atmosphere. Therefore the effective pseudo-Roche limiting surface is modified and a crisis may occur for a smaller (stellar radius)/(companion distance) ratio. Perhaps the result would mimic rapid rotation by a single isolated star.

Maeder: I see a number of difficulties in binary models for explaining the LBV phenomenon: (1) It would seem difficult to keep $L=$ constant during the accretion process, among binaries with different orbital parameters and mass ratios.

(2) If they are all binaries, why don't we see a clock in any of them?

(3) In view of the diverse binary parameters, why would binary models be confined to the left of the H-D limit? Binaries of all masses exist but the LBV phenomenon is restricted to a limited part of the H-R diagram.

(4) How can the well-known short-period pulsations survive in such distorted stars?

(5) I do not see tidal mixing, which could seriously change the overall picture, included in your models.

(6) Finally, even if some LBV's are binaries, we do not need the binary hypothesis; the upper-right part of the H-R diagram is forbidden to static models anyway.

Gallagher: These are all interesting points. Let me respond briefly to each point in order. (1) The accretion luminosity is minor in the preferred models where binary processes supply main the enhanced mass loss. Thus, while variations in accretion rates will be needed to modulate the mass-loss rate, these should not lead to perceptible luminosity variations. (2) When strong interactions occur, it is of ten hard to see the clocks even in known binaries (e.g., dwarf novae and symbiotic stars). (3) In this conceptual model, the combination of near-Eddington luminosities and destabilization by binary interactions produces an LBV. Quantitative boundaries need to be defined for these processes. (4) Perhaps the pulsations take place in the common envelope, as seems to occur in post-maximum classical novae. (5) I agree that the conconceptual picture presented here should be confirmed via quantitative models. (6) The existence of short-period, high-mass binaries containing compact stars could be viewed as evidence that binary interactions do play a role in determining the properties of massive stellar populations. The question in my mind is to find the relative importance of binary versus single-star evolutionary paths to the LBV's. 